\title{
Scaling Limit for Interacting Ornstein-Uhlenbeck Processes *
}

\author{
Stefano Olla and S. R. S. Varadhan \\ Courant Institute of Mathematical Sciences, New York University, 251 Mercer Street, \\ New York, NY 10012, USA
}

Received February 26, 1990; in revised form July 26, 1990

\begin{abstract}
The problem of describing the bulk behavior of an interacting system consisting of a large number of particles comes up in different contexts. See for example [1] for a recent exposition. In [4] one of the authors considered the case of interacting diffusions on a circle and proved that the density of particles evolves according to a nonlinear diffusion equation. The interacting particles evolved according to a generator that was symmetric in equilibrium. In this article we consider interacting Ornstein-Uhlenbeck processes. Here the diffusion generator is not symmetric relative to the equilibrium and the earlier methods have to be modified considerably. We use some ideas that were employed in [3] to extend the central limit theorem from the symmetric to nonsymmetric cases.
\end{abstract}

\section{The Model and Its Macroscopic Equation}

Let $S$ be the circle of circumference 1. For each positive integer $N$ we consider a system of $N$ interacting particles with positions on $S$ and velocities in $R$. The system is described by the following stochastic differential equations in phase space $(\underline{x}, \underline{v})=\left\{\left(x_{1}, v_{1}\right),\left(x_{2}, v_{2}\right), \ldots,\left(x_{N}, v_{N}\right)\right\}$,

$$
\begin{aligned}
d x_{i}(t)= & N v_{i}(t) d t \\
d v_{i}(t)= & -N^{2} \sum_{j \neq i} 2 V^{\prime}\left(N\left(x_{i}(t)-x_{j}(t)\right)\right) d t \\
& -\frac{N^{2}}{2} v_{i}(t) d t+N d w_{i}(t)
\end{aligned}
$$

for $i=1,2, \ldots, N ; 0 \leqq t \leqq T$. Here $\left\{w_{i}(t), i=1,2, \ldots, N\right\}$ are $N$ independent Wiener processes and $V$ is an even function on $R$ with compact support describing a pair interaction.

* This research is supported in part by the National Science Foundation, grant nos. DMS 8901682 and DMS-88-06727 
We make the following assumptions on $V$ :

(i) $V \geqq 0, V(0)>0$ and $V$ has compact support (super stability).

(ii) $V$ is once continuously differentiable.

(iii) $\psi(z)=-z V^{\prime}(z) \geqq 0$ (Repulsive Interaction).

The generator of the Markov Process $(\underline{x}(t), \underline{v}(t))$ on $(S \times R)^{N}$ is given by

$$
L_{N}=S_{N}+J_{N} \text {, }
$$

where

$$
\begin{aligned}
& S_{N}=\frac{N^{2}}{2} \sum_{i=1}^{N}\left(\frac{\partial^{2}}{\partial v_{i}^{2}}-v_{i} \frac{\partial}{\partial v_{i}}\right), \\
& J_{N}=N \sum_{i=1}^{N}\left\{v_{i} \frac{\partial}{\partial x_{i}}-N \sum_{j \neq i} 2 V^{\prime}\left(N\left(x_{i}-x_{j}\right)\right) \frac{\partial}{\partial v_{i}}\right\} .
\end{aligned}
$$

The "equilibrium" measure on $(S \times R)^{N}$ for $L_{N}$ is given by

$$
d \mu_{N}(\underline{x}, \underline{v})=\frac{1}{Z_{N}} e^{-\frac{1}{2} \sum_{i=1}^{N} v^{2}-\sum_{i \neq j}^{N} V\left(N\left(x_{i}-x_{j}\right)\right)} d^{N x} d^{N} \underline{v},
$$

where $Z_{N}$ is the normalizing constant. We note that $S_{N}$ and $J_{N}$ are respectively the symmetric and antisymmetric part of $L_{N}$ with respect to $\mu_{N}$.

We will assume that the initial distribution of the process has a density $f_{N}^{0}(\underline{x}, \underline{v})$ with respect to $\mu_{N}$ and it satisfies the following entropy bound:

$$
H_{N}\left(f_{N}^{0}\right)=\int f_{N}^{0}(\underline{x}, \underline{v}) \log f_{N}^{0}(\underline{x}, \underline{v}) d \mu_{N}(\underline{x}, \underline{v}) \leqq C N
$$

for some suitable constant $C$.

The empirical distribution of the process at time $t$ is the probability measure on $S$ defined by

$$
\alpha_{N}(t, d \theta)=\frac{1}{N} \sum_{i=1}^{N} \delta_{x_{i}(t)}(d \theta)
$$

We view $\alpha_{N}$ as an element of $C\left[[0, T], M_{1}(S)\right]$, the space of continuous functions on $[0, T]$ with values in the space $M_{1}(S)$ of probability measures on $S$, endowed with the weak topology. We denote by $Q_{N}$ the distribution of $\alpha_{N}$ on $C[[0, T]$, $\left.M_{1}(S)\right]$. The aim of this article is to study the limiting behavior of $Q_{N}$. The main result is that as $N \rightarrow \infty, Q_{N}$ will concentrate around a single trajectory which is the solution of a nonlinear diffusion equation.

Theorem 1.1 (Hydrodynamic Limit). Assume that $H_{N}\left(f_{N}^{0}\right) \leqq C N$ and that there exists a function $m_{0}(\theta)$ such that $m_{0}(\theta) \geqq 0, \int m_{0}(\theta) d \theta=1$ and for every $J: S \rightarrow R$ which is smooth and every $\delta>0$,

$$
\lim _{N \rightarrow \infty} \int_{A_{N}, \delta, J} f_{N}^{0}(\underline{x}, \underline{v}) d \mu_{N}(\underline{x}, \underline{v})=0,
$$

where

$$
A_{N, \delta, J}=\left\{(\underline{x}, \underline{v}) \in(S \times R)^{N}:\left|\frac{1}{N} \sum_{i=1}^{N} J\left(x_{i}\right)-\int J(\theta) m_{0}(\theta) d \theta\right| \geqq \delta\right\},
$$


then $Q=\lim _{N \rightarrow \infty} Q_{N}$ exists and it is concentrated on the trajectory of the form $\mu(t, d \theta)$ in $C\left[[0, T], M_{1}(S)\right]$ satisfying

(i) $\mu(t, d \theta)=m(t, \theta) d \theta$ for all $t$.

(ii) $\int_{0}^{T} d t \int_{S} d \theta[m(t, \theta)]^{3}<\infty$.

(iii) $\int_{0}^{T} d t \int_{S} \frac{1}{m(t, \theta)}\left[\frac{\partial}{\partial \theta} P(m(t, \theta))\right]^{2} d \theta<\infty$.

(iv) $m(t, \theta)$ is a weak solution of the equation

$$
\frac{\partial m}{\partial t}=2 \frac{\partial^{2} P(m(\theta, t))}{\partial \theta^{2}}
$$

with initial condition $m(t, \theta)$ at $t=0$ given by $m_{0}(\theta)$. Furthermore the weak solution (1.6) satisfying (ii) and (iii) above is unique. The function $P(\cdot)$ is the thermodynamic function "pressure" which is defined in Sect. 2.

This theorem will be proved in Sect. 5 using the basic results of Sects. 3 and 4. The main idea is the following.

Let $f_{N}^{t}(\underline{x}, \underline{v})$ be the solution of the forward equation

$$
\frac{\partial f_{N}^{t}}{\partial t}=L_{N}^{*} f_{N}^{t}
$$

with initial condition $f_{N}^{0}$, where $L_{N}^{*}$ is adjoint of $L_{N}$ with respect to the measure $\mu_{N}$. The core of the problem is to prove certain local ergodic properties of the time average

$$
\bar{f}_{N}(\underline{x}, \underline{v})=\frac{1}{T} \int_{0}^{T} f_{N}^{t}(\underline{x}, \underline{v}) d t .
$$

These local ergodic properties will be established in Sect. 4 and are based on certain bounds on entropy and its rate of change. An elementary computation yields

$$
\frac{d}{d t} H_{N}\left(f_{N}^{t}\right)=-\frac{N^{2}}{2} \int \frac{1}{f_{N}^{t}} \sum_{i=1}^{N}\left(\frac{\partial f_{N}^{t}}{\partial v_{i}}\right)^{2} d \mu_{N} \leqq 0 .
$$

This implies immediately that $H_{N}\left(f_{N}^{t}\right) \leqq C N$ for all $t \geqq 0$. Defining for any density $f$ relative to $\mu_{N}$

we have

$$
I_{N}(f)=\frac{1}{2} \int \frac{1}{f} \sum_{i=1}^{N}\left(\frac{\partial f}{\partial v_{i}}\right)^{2} d \mu_{N}
$$

$$
\int_{0}^{T} I_{N}\left(f_{N}^{t}\right) d t=-\frac{1}{N^{2}}\left(H_{N}\left(f_{N}^{T}\right)-H_{N}\left(f_{N}^{0}\right)\right) \leqq \frac{C}{N} .
$$

Since both $I_{N}(f)$ and $H_{N}(f)$ are convex functionals of $f$ we have

$$
\begin{aligned}
& I_{N}\left(\bar{f}_{N}\right) \leqq \frac{C}{T N}, \\
& H_{N}\left(\bar{f}_{N}\right) \leqq C N .
\end{aligned}
$$




\section{Canonical and Grand Canonical Gibbs Measures}

Let $\mathscr{X}$ denote the set of all locally finite configurations of particles on the real line $R$. By a locally finite configuration we mean a countable subset of $R$ with no accumulation points in $R$. For each value of a real parameter $\lambda$ called the chemical activity the Grand Canonical Partition function in a finite interval $\Lambda$ is defined as

$$
Z(\lambda, \Lambda)=\sum_{n \geqq 0} \frac{e^{n \lambda}}{n !} \int_{\lambda^{n}} \exp \left[-\sum_{i \neq j} V\left(q_{i}-q_{j}\right)\right] d q_{1} \ldots d q_{n} .
$$

The free energy as a function $F(\lambda)$ of $\lambda$ is defined by

$$
F(\lambda)=\lim _{\Lambda \uparrow R} \frac{1}{|\Lambda|} \log Z(\lambda, \Lambda) .
$$

By the assumptions made on the interaction $V$, the limit (2.2) exists and defines a convex function $F(\lambda)$ of $\lambda$. In our case since the dimension is 1 , the function $F(\lambda)$ is continuously differentiable with a derivative

$$
\varrho(\lambda)=\frac{d F(\lambda)}{d \lambda} .
$$

The function $\varrho(\lambda)$ is continuous and strictly increasing with $\varrho(\lambda) \rightarrow 0$ as $\lambda \rightarrow-\infty$ and $\varrho(\lambda) \rightarrow+\infty$ as $\lambda \rightarrow+\infty$. The inverse $\lambda=\lambda(\varrho)$ exists as a continuous increasing function and

$$
P(\varrho)=F(\lambda(\varrho))
$$

defines the pressure $P(\varrho)$ as a continuous, strictly increasing function of $\varrho$.

The canonical Gibbs measure in a finite interval $\Lambda$ with particle number $n$ and external boundary condition $\omega$ is a probability measure $\mu_{n, A}^{\omega}$ on configurations in $\Lambda$ with $n$ points or equivalently, a symmetric probability measure on $\Lambda^{n}$. The external boundary condition is a configuration on $\Lambda^{c}$. We define

$$
d \mu_{n, A}^{\omega}=\frac{1}{n !}\left[Z_{n, A}^{\omega}\right]^{-1} \exp \left[-\sum V\left(q_{i}-q_{j}\right)-2 \sum V\left(q_{i}-y_{\alpha}\right)\right] d q_{1} \ldots d q_{n},
$$

where $Z_{n, A}^{\omega}$ is the normalization constant,

$$
Z_{n, \Lambda}^{\omega}=\frac{1}{n !} \int_{\Lambda^{n}} \exp \left[-\sum V\left(q_{i}-q_{j}\right)-2 \sum V\left(q_{i}-y_{\alpha}\right)\right] d q_{1} \ldots d q_{N} .
$$

The Grand Canonical Gibbs measure on a finite interval $\Lambda$ with activity $\lambda$ is a convex combination of $\mu_{n, \Lambda}^{\omega}$ with special weights. We define

where

$$
\hat{\mu}_{\lambda, \Lambda}^{\omega}=\left[\hat{Z}_{\lambda, \Lambda}^{\omega}\right]^{-1} \sum_{n=0}^{\infty} e^{\lambda n} Z_{n, \Lambda}^{\omega} \mu_{n, \Lambda}^{\omega},
$$

$$
\hat{Z}_{\lambda, \Lambda}^{\omega}=\sum_{n=0}^{\infty} e^{\lambda n} Z_{n, \lambda}^{\omega}
$$

The Grand Canonical Gibbs measure on the infinite line with activity $\lambda$ is a point process $\mu_{\lambda}$ on the configuration space $\mathscr{X}$ such that for every finite interval $\Lambda$ the conditional distribution of the configuration in $\Lambda$ given the configuration $\omega$ in the exterior $\Lambda^{c}$, is given by $\hat{\mu}_{\lambda, \Lambda}^{\omega}$ for almost all $\omega$. 
One of the important aspects of the absence of phase transition in our one dimensional system is the following theorem. It is stated here without proof. A sketch of the proof can be found in the appendix of [4].

Theorem 2.1. For each $\lambda$, there is exactly one Grand Canonical Gibbs measure $\mu_{\lambda}$ on $\mathscr{X}$ corresponding to the activity $\lambda$. It is a stationary point process with density $\varrho=\varrho(\lambda)$ given by (2.3). If $\mu_{n, \Lambda}^{\omega}$ are canonical Gibbs measures such that $\Lambda \uparrow R$ and $\frac{n}{|\Lambda|} \rightarrow \varrho$, then for any continuous bounded local function $H\left(\omega^{\prime}\right)$ on the configuration space,

$$
\lim _{\substack{n /|\Lambda| \rightarrow \mid \rightarrow \infty \\ \Lambda \uparrow \mathbb{R}}} \sup _{\omega} \mu_{n, \Lambda}^{\omega}\left[\left|\frac{1}{|\Lambda|} \int_{\Lambda} H\left(\omega_{x}^{\prime}\right) d x-\hat{H}(\varrho)\right| \geqq \delta\right]=0 .
$$

Here $\hat{H}(\varrho)=E^{\mu_{\lambda}}\left[H\left(\omega^{\prime}\right)\right]$ with $\lambda$ chosen so that $\varrho(\lambda)=\varrho$. The configuration $\omega^{\prime}$ translated in space by $x$ is denoted by $\omega_{x}^{\prime}$.

The correlation measures $R_{\lambda}^{(k)}\left(d z_{1}, \ldots, d z_{k}\right)$ are defined so that

$$
E^{\mu_{\lambda}}\left[\sum_{\left(z_{1}, \ldots, z_{k}\right)} f\left(z_{1}, \ldots, z_{k}\right)\right]=\int_{R^{k}} f\left(z_{1}, \ldots, z_{k}\right) R_{\lambda}^{(k)}\left(d z_{1}, \ldots, d z_{k}\right) .
$$

On the left-hand side the summation is over all $k$-tuples $\left(z_{1}, \ldots, z_{k}\right)$ that belong to the configuration. $f\left(z_{1}, \ldots, z_{k}\right)$ is assumed to be a bounded continuous function with compact support. $R_{\lambda}^{(k)}\left(d z_{1}, \ldots, d z_{k}\right)$ is a $\sigma$-finite measure on $R^{k}$ invariant with respect to diagonal translations $z_{1}, \ldots, z_{k} \rightarrow z_{1}+a, \ldots, z_{k}+a$. In particular

$$
R_{\lambda}^{(1)}(d z)=\varrho(\lambda) d z .
$$

We have the identity proved in the appendix of [4].

Theorem 2.2. For every $\lambda$ and $\varrho$ related by $\varrho=\varrho(\lambda)$,

$$
\varrho+\iint \psi(x-y) h(y) R_{\lambda}^{(2)}(d x, d y)=P(\varrho),
$$

where $h(y)$ is any function which has compact support with $\int_{-\infty}^{\infty} h(y) d y=1$.

\section{Some Estimates Based on Entropy and the Dirichlet Form}

In this section our aim is to obtain some elementary bounds based on the inequalities

and

$$
H_{N}\left(f_{N}\right) \leqq A N
$$

$$
I_{N}\left(f_{N}\right) \leqq \frac{C}{N}
$$

for an arbitrary density $f_{N}(\underline{x}, \underline{v})$ relative to $\mu_{N}$.

Theorem 3.1. Let $f_{N}$ satisfy (3.1). Then

$$
\int|\underline{\mid}|^{2} f_{N} d \mu_{N} \leqq N(\log \sqrt{2}+A) .
$$


(ii) For any function $\phi$ on $C(R)$ with compact support there exists a constant $C^{\prime}$ such that for all $N$ and $\lambda \leqq 1$,

Proof.

$$
\int \lambda \sum_{i, j} \phi\left(\lambda N\left(x_{i}-x_{j}\right)\right) f_{N} d \mu_{N} \leqq C^{\prime} N .
$$

$$
\text { (i) } \begin{aligned}
\frac{1}{4} \int\left[\sum_{i=1}^{N} v_{i}^{2}\right] f_{N} d \mu_{N} & \leqq \\
& \log \int \exp \left[\frac{1}{4} \sum v_{i}^{2}\right] d \mu_{N}+H_{N}\left(f_{N}\right) \\
& =N(\log \sqrt{2}+A) .
\end{aligned}
$$

(ii) The proof can be found in Lemma 4.2 of [4].

In the following we will denote the Gaussian product distribution on $R^{N}$ by

$$
G_{N}(d \underline{v})=\frac{1}{(2 \pi)^{N / 2}} e^{-(1 / 2)|v|^{2}} d^{N} \underline{v},
$$

and the equilibrium distribution on $S^{N}$ by

$$
d \mu_{N}^{*}(\underline{x})=\frac{1}{Z_{N}^{*}} \exp \left(-\sum_{i \neq j} V\left(N\left(x_{i}-x_{j}\right)\right) d^{N} \underline{x}\right.
$$

so that $d \mu_{N}(\underline{x}, \underline{v})=d G_{N}(\underline{v}) \cdot \mu_{N}^{*}(\underline{x})$. Given a probability density $f_{N}(x, v)$ relative to $\mu_{N}$, we denote by $f_{N}(\underline{v} \mid \underline{x})$ the density of the conditional distribution of $\underline{v}$ given $x$, relative to $G_{N}(d \underline{v})$. By the logarithmic Sobolev inequality (see [1] for a proof) for any configuration $\underline{x}$ we have

$$
\int f_{N}(\underline{v} \mid \underline{x}) \log f_{N}(\underline{v} \mid \underline{x}) G_{N}(d \underline{v}) \leqq 2 \int \frac{1}{f_{N}(\underline{v} \mid \underline{x})}\left|\nabla_{v} f_{N}(\underline{v} \mid \underline{x})\right|^{2} G_{N}(d \underline{v}) .
$$

If we denote the marginal of $f_{N}(\underline{x}, \underline{v})$ by

$$
f_{N}^{*}(\underline{x})=\int f_{N}(\underline{x}, \underline{v}) G_{N}(d \underline{v}),
$$

then by a simple calculation

$$
\begin{aligned}
& \int_{S^{N}} f_{N}^{*}(\underline{x}) d \mu_{N}^{*}(\underline{x}) \int_{R^{N}} f_{N}(\underline{v} \mid \underline{x}) \log f_{N}(\underline{v} \mid \underline{x}) G_{N}(d \underline{v}) \\
& \leqq 2 \int \frac{1}{f_{N}(\underline{x}, \underline{v})}\left|\nabla_{v} f_{N}(\underline{x}, \underline{v})\right|^{2} d \mu_{N}(\underline{x}, \underline{v})=4 I_{N}\left(f_{N}\right) .
\end{aligned}
$$

We have therefore proved

Lemma 3.2. Let $f_{N}$ be such that $I_{N}\left(f_{N}\right) \leqq C / N$. Then the conditional density $f_{N}(\underline{v} \mid \underline{x})$ satisfies

$$
\int f_{N}^{*}(\underline{x}) d \mu_{N}^{*}(\underline{x}) \int G_{N}(d \underline{v}) f_{N}(\underline{v} \mid \underline{x}) \log f_{N}(\underline{v} \mid \underline{x}) \leqq \frac{4 C}{N} .
$$

\section{Local Equilibrium Distributions}

We would like to show that, relative to the probability distribution $\bar{f}_{N} d \mu_{N}$, "average microscopic quantities" like $\frac{1}{N} \sum \psi\left(N\left(x_{i}-x_{j}\right)\right) J\left(x_{i}\right)$ are close, for large $N$, to the expression involving only the macroscopic density function. 
The approach we use here is similar to the one used in [4], but the information we have on $\bar{f}_{N}$ here are different. In [4] two basic estimates available on $\bar{f}_{N}$ are used,

and

$$
\int \bar{f}_{N} \log \bar{f}_{N} d \mu_{N} \leqq B N
$$

$$
\frac{1}{2} \int \frac{1}{\bar{f}_{N}} \sum_{i=1}^{N}\left(\frac{\partial \bar{f}_{N}}{\partial x_{i}}\right)^{2} d \mu_{N} \leqq D N .
$$

In our case we do not have (4.2) but instead have

$$
\frac{1}{2} \int \frac{1}{\bar{f}_{N}} \sum_{i=1}^{N}\left(\frac{\partial \bar{f}_{N}}{\partial v_{i}}\right)^{2} d \mu_{N} \leqq \frac{D}{N} .
$$

As we saw in the previous section, by the logarithmic Sobolev inequality this will tell us that the distribution of the velocities conditioned on the positions are all very close to the equilibrium. But this says nothing about the distribution of positions. To infer from this that the local position distributions are close to equilibrium distributions and that the macroscopic density has no unnecessary fluctuations, we have to actually use information that $\bar{f}_{N}$ are time averages of the solution to the forward equation.

Consider a function $\Phi(\omega)$ on the configuration of points in $R$ which is bounded, continuous and localized in some finite interval $[-l, l]$. For any given $x \in S$ and any $\underline{x} \in S^{N}$ we can consider the configuration $\omega_{N}^{x}$ on the line

$$
\omega_{N}^{x}=\left\{N\left(x_{i}-x\right):\left|x_{i}-x\right|<\frac{1}{4}\right\} .
$$

If $N$ is sufficiently large $\Phi\left(\omega_{N}^{x}\right)$ makes sense and we are interested in the quantity

$$
\xi_{N}(\underline{x})=\int_{S} \Phi\left(\omega_{N}^{x}\right) J(x) d x,
$$

let $h(x)$ be a nonnegative smooth function with $\int h(x) d x=1$, supported on $\left[-\frac{1}{2}, \frac{1}{2}\right]$. For $\lambda>1$, we define

$$
\begin{aligned}
\varrho_{\lambda}(x) & =\frac{\lambda}{N} \sum_{i=1}^{N} h\left(\lambda\left(x_{i}-x\right)\right) \\
& =\left(h_{\lambda} * \alpha_{N}\right)(x),
\end{aligned}
$$

where $h_{\lambda}(x)=\lambda h(\lambda x)$ and $\alpha_{N}(d x)=\frac{1}{N}\left[\delta_{x_{1}}+\ldots \delta_{x_{N}}\right]$ is the empirical distribution of the configuration $\left(x_{1}, \ldots, x_{N}\right)$ on $S$. We denote by $\hat{\Phi}(\varrho)$ the expected value

$$
E^{\mu_{e}}[\Phi(\omega)]=\hat{\Phi}(\varrho),
$$

where $\mu_{\varrho}$ is the Gibbs measure with density $\varrho$ or activity $\lambda=\lambda(\varrho)$. If we define

$$
\eta_{N, \lambda}(\underline{x})=\int_{S} \hat{\Phi}\left(\varrho_{\lambda}(x)\right) J(x) d x,
$$

then we want to prove

\section{Theorem 1.}

$$
\lim _{\lambda \rightarrow \infty} \limsup _{N \rightarrow \infty} E^{\bar{f}_{N}}\left|\xi_{N}(\underline{x})-\eta_{N, \lambda}(x)\right|=0 .
$$

As in [4] we establish Theorem 1.1 in two steps. 
Theorem 4.2.

$$
\lim _{\varepsilon \rightarrow 0} \limsup _{N \rightarrow \infty} E^{\bar{f}_{N}}\left|\xi_{N}(\underline{x})-\eta_{N, N_{\varepsilon}}(x)\right|=0
$$

Theorem 4.3.

$$
\lim _{\substack{\varepsilon \rightarrow 0 \\ \lambda \rightarrow \infty}} \limsup _{N \rightarrow \infty} E^{\bar{f}_{N}} \int_{S}\left|\varrho_{\lambda}(x)-\varrho_{\varepsilon N}(x)\right| d x=0 .
$$

Let $\hat{f}_{N}(\underline{x}, \underline{v})=\int_{S} \bar{f}_{N}(\underline{x}+a, v) d a$. Then by convexity we have that $\hat{f}_{N}$ satisfies

$$
\begin{gathered}
H_{N}\left(\hat{f}_{N}\right) \leqq A N, \\
I_{N}\left(\hat{f}_{N}\right) \leqq \frac{C}{N} .
\end{gathered}
$$

In exactly the same way as in [4] the proof of Theorem 4.2 can be reduced to

\section{Theorem 4.4.}

$$
\lim _{\varepsilon \rightarrow 0} \limsup _{N \rightarrow \infty} E^{\bar{f}_{N}}\left|\frac{N \varepsilon}{2} \int_{|x| \leqq 1 / N \varepsilon} \Phi\left(\omega_{N}^{x}\right) d x-\hat{\Phi}\left(\varrho_{N \varepsilon}(0)\right)\right|=0 .
$$

Proof of Theorem 4.4. Both $\int_{|y| \leqq 1 / N \varepsilon} \Phi\left(\omega_{y}^{N}\right) d y$ and $\varrho_{N \varepsilon}(0)$ depend only on the configuration $x$ in the interval $\left[-\frac{1}{\varepsilon N}-\frac{l}{N}, \frac{1}{\varepsilon N}+\frac{l}{N}\right]$ centered around the origin of $S$. Here the function $\Phi$ is assumed to depend only on the configuration in $[-l, l]$. If we project $\hat{f}_{N} d \mu_{N}$ onto configurations on this interval and expand the interval by a factor $N$, we will get a point process $\hat{v}_{N, \varepsilon}$ on the interval $\left[-\frac{1}{\varepsilon}-l, \frac{1}{\varepsilon}+l\right]$. All we have to prove is that

$$
\lim _{\varepsilon \rightarrow 0} \limsup _{N \rightarrow \infty} E^{\hat{v}_{N, \varepsilon}}\left|\frac{\varepsilon}{2} \int_{-1 / \varepsilon}^{1 / \varepsilon} \Phi\left(\omega^{x}\right) d x-\hat{\Phi}\left(\varrho_{\varepsilon}(0)\right)\right|=0,
$$

where $\omega^{x}$ is the configuration $\omega$ translated by $x$ in space. Since the density of particles is 1 under $\hat{f}_{N} d \mu_{N}, \hat{v}_{N, \varepsilon}$ is compact and we denote by $\hat{\mathscr{B}}_{\varepsilon}$ the set of all limit points of $\hat{v}_{N, \varepsilon}$ as $N \rightarrow \infty$. We have to prove that

$$
\lim _{\varepsilon \rightarrow 0} \sup _{\nu \in \mathscr{\not}_{\varepsilon}} E^{\nu}\left[\left|\frac{\varepsilon}{2} \int_{-1 / \varepsilon}^{1 / \varepsilon} \Phi\left(\omega^{x}\right) d x-\hat{\Phi}\left(\varrho_{\varepsilon}(0)\right)\right|\right]=0 .
$$

Let $\mu_{n, l}^{\omega}$ be the Gibbs measure on $[-l, l]$ with exterior boundary condition $\omega$ and $n$ particles inside $[-l, l]$. We denote by $\Gamma_{l}$ the convex hull of these measures, and by $\Gamma_{l}^{(1)}$ those for which expected particle density in $[-l, l]$ is at most 1 . Then according to Theorem 2.1,

$$
\lim _{k \rightarrow \infty} \sup _{v \in \Gamma_{l+k}^{(1)}} E^{\nu}\left[\left|\frac{1}{2 k} \int_{-k}^{k} \Phi\left(\omega^{x}\right) d x-\hat{\Phi}\left(\varrho_{1 / k}(0)\right)\right|\right]=0,
$$

and we therefore need only establish the lemma

Lemma 4.5. For every $l$ and $\varepsilon$

$$
\widehat{\mathscr{B}}_{\varepsilon} \subset \Gamma_{(1 / \varepsilon)+l}^{(1)}
$$


Proof. Let $c_{0}$ be the range of the interaction $V$, i.e. $V(x)=0$ if $|x| \geqq c_{0}$. Let us consider the limiting point process on the interval $\left[-\frac{1}{\varepsilon}-l-c_{0}, \frac{1}{\varepsilon}+l+c_{0}\right]$, i.e. any distribution $v \in \widehat{\mathscr{B}}_{\varepsilon}$, where $1 / \varepsilon^{\prime}+l=1 / \varepsilon+l+c_{0}$. We want to show that $v \in \Gamma_{l+(1 / \varepsilon)}^{(1)}$.

Consider the function

$$
u_{N}(\underline{x}, \underline{v})=\sum_{i=1}^{N} v_{i} \Phi\left(\omega_{N}^{0}\right) g\left(N x_{i}\right),
$$

where $\Phi$ is any function of the configuration of points in $\left[-l-c_{0}, l+c_{0}\right]$ which is bounded continuous and differentiable, and $g$ is any positive continuous function with compact support contained in $[-l, l]$. By direct computation we have

$$
\begin{aligned}
\sum_{i, j} 2 V^{\prime}\left(N\left(x_{i}-x_{j}\right)\right) & \Phi\left(\omega_{N}^{0}\right) g\left(N\left(x_{i}\right)\right)-\sum_{i, j} \frac{1}{N} v_{i} v_{j} \frac{\partial}{\partial x_{i}}\left(\Phi\left(\omega_{N}^{0}\right) g\left(N\left(x_{j}\right)\right)\right) \\
& =u_{N}(\underline{x}, \underline{v})-\frac{1}{N^{2}}\left(L_{N} u_{N}\right)(\underline{x}, \underline{v}) .
\end{aligned}
$$

On integrating by parts:

$$
\begin{aligned}
&\left|\int u_{N}(\underline{x}, \underline{v}) \hat{f}_{N}(\underline{x}, \underline{v}) d \mu_{N}\right|=\left|\int_{S} d a \int u_{N}(\underline{x}+a, \underline{v}) \bar{f}_{N}(\underline{x}, \underline{v}) d \mu_{N}\right| \\
&=\left|\int_{S} d a \int \Phi\left(\omega_{N}^{a}\right) \sum_{i=1}^{N} g\left(N\left(x_{i}-a\right)\right) \frac{\partial \bar{f}_{N}}{\partial v_{i}} d \mu_{N}\right| \\
& \leqq\|\Phi\|_{\infty}\left(\int \sum_{i} g\left(N x_{i}\right) \hat{f}_{N} d \mu_{N}\right)^{1 / 2} \\
& \times\left(\int_{S} d a \int \frac{1}{\bar{f}_{N}} \sum\left(\frac{\partial \bar{f}_{N}}{\partial v_{i}}\right)^{2} g\left(N\left(x_{i}-a\right)\right) d \mu_{N}\right)^{1 / 2} \\
& \leqq\|\Phi\|_{\infty}\left(\frac{C}{N} \cdot \int \frac{1}{\bar{f}_{N}} \sum\left(\frac{\partial \bar{f}_{N}}{\partial v_{i}}\right)^{2} d \mu_{N}\right)^{1 / 2} \leqq \frac{C^{\prime}}{N} .
\end{aligned}
$$

If $g_{N}$ is any probability density with respect to $\mu_{N}$ and $\hat{g}_{N}$ is its average

$$
\begin{aligned}
\int_{S} g_{N}(\underline{x}+a, \underline{v}) d a & =\hat{g}_{N}(\underline{x}, \underline{v}) \\
\int u_{N}(\underline{x}, \underline{v}) \hat{g}_{N} d \mu_{N} & =\int_{S} \int u_{N}(\underline{x}+a, \underline{v}) g_{N}(\underline{x}, \underline{v}) d \mu_{N} d a \\
& \leqq\|\Phi\|_{\infty}\left(\int \frac{1}{N} \sum\left|v_{i}\right| g_{N}(\underline{x}, \underline{v}) d \mu_{N}\right) \\
& \leqq\|\Phi\|_{\infty}\left(\int \frac{1}{N} \sum\left|v_{i}\right|^{2} g_{N}(\underline{x}, \underline{v}) d \mu_{N}\right)^{1 / 2} \\
& \leqq\|\Phi\|_{\infty}\left[\frac{4}{N} \log \int \exp \left[\frac{1}{4} \sum v_{i}^{2}\right] d \mu_{N}+\frac{4}{N} H\left(g_{N}\right)\right]^{1 / 2} \\
& \leqq C^{\prime}\|\Phi\|_{\infty},
\end{aligned}
$$


provided $H\left(g_{N}\right) \leqq C N$. If we now use the explicit formula $\hat{f}_{N}=\frac{1}{T} \int_{0}^{T} \hat{f}_{N}^{t} d t$ and calculate

$$
\begin{aligned}
\int L_{N} u_{N} \hat{f}_{N} d \mu_{N} & =\frac{1}{T} \int_{0}^{T} \int u_{N} \frac{\partial \hat{f}_{N}^{t}}{\partial t} d t d \mu_{N} \\
& =\frac{1}{T}\left[\int u_{N} \hat{f}_{N}^{T} d \mu_{N}-\int u_{N} \hat{f}_{N}^{0} d \mu_{N}\right]
\end{aligned}
$$

then

$$
\left|\int L_{N} u_{N} \hat{N}_{N} d \mu_{N}\right| \leqq 2 C^{\prime}\|\Phi\|_{\infty}
$$

because $H\left(\hat{f}_{N}^{T}\right) \leqq H\left(\hat{f}_{N}^{0}\right) \leqq C N$.

We have therefore established

$$
\begin{gathered}
\lim _{N \rightarrow \infty} E^{\hat{f}_{N}}\left[\sum_{i, j} 2 V^{\prime}\left(N\left(x_{i}-x_{j}\right)\right) \Phi\left(\omega_{N}^{0}\right) g\left(N x_{i}\right)\right. \\
\left.-\frac{1}{N} \sum_{i, j} v_{i} v_{j} \frac{\partial}{\partial x_{i}}\left(\Phi\left(\omega_{N}^{0}\right) g\left(N x_{j}\right)\right)\right]=0 .
\end{gathered}
$$

Let us define the following $N \times N$ matrix

$$
F_{i j}(\underline{x})=\frac{1}{N} \frac{\partial}{\partial x_{i}}\left(\Phi\left(\omega_{N}^{0}\right) g\left(N x_{j}\right)\right) .
$$

Because of the scaling $\left\{F_{i j}(\underline{x})\right\}$ are uniformly bounded. Then by the entropy estimates of Sect. 3,

$$
\begin{aligned}
\int \frac{1}{N} & \sum_{i=1}^{N}\left(v_{i}^{2}-1\right) F_{i i}(\underline{x}) \hat{f}_{N}(\underline{x}, \underline{v}) d \mu_{N} \\
\leqq & \int \hat{f}_{N}^{*}(\underline{x}) d \mu_{N}^{*}(\underline{x})\left\{\log \int \exp \left[\frac{1}{N} \sum\left(v_{i}^{2}-1\right) F_{i i}(\underline{x})\right] G_{N}(d \underline{v})\right. \\
& \left.+\int \hat{f}_{N}(\underline{v} \mid \underline{x}) \log \hat{f}_{N}(\underline{v} \mid \underline{x}) G_{N}(d \underline{v})\right\} \\
\leqq & \int \hat{f}_{N}^{*}(\underline{x}) d \mu_{N}^{*}(\underline{x})\left\{\log \int \exp \left[\frac{1}{N} \sum\left(v_{i}^{2}-1\right) F_{i i}(\underline{x})\right] G_{N}(d \underline{v})\right\}+\frac{2 C}{T N} .
\end{aligned}
$$

By an elementary calculation, essentially because of the law of large numbers we can conclude that the last term goes to zero with $N$. Therefore

$$
\limsup _{N \rightarrow \infty} \frac{1}{N} \sum\left[v_{i}^{2}-1\right] F_{i i}(\underline{x}) \hat{f}_{N}(\underline{x}, \underline{v}) d \mu_{N} \leqq 0 .
$$

Since the same argument works with change of sign we actually have

$$
\lim _{N \rightarrow \infty}\left|\frac{1}{N} \int \sum\left(v_{i}^{2}-1\right) F_{i i}(\underline{x}) \hat{f}_{N}(\underline{x}, \underline{v}) d \mu_{N}\right|=0 .
$$


Using an exactly similar argument on the off diagonal elements

$$
\begin{aligned}
& \frac{1}{N} \int \sum_{i \neq j} v_{i} v_{j} F_{i j}(\underline{x}) \hat{f}_{N}(\underline{x}, \underline{v}) d \mu_{N} \\
& \quad \leqq \int \hat{f}_{N}^{*}(\underline{x}) d \mu_{N}^{*}\left\{\frac{1}{\sigma} \log \int \exp \left[\frac{\sigma}{N} \sum_{i \neq j} v_{i} v_{j} F_{i j}(\underline{x})\right] G_{N}(d \underline{v})+\frac{2 C}{\sigma N T}\right\} .
\end{aligned}
$$

We can estimate by direct calculation

$$
\begin{aligned}
\int \exp \left[\frac{\sigma}{N} \sum_{i \neq j} v_{i} v_{j} F_{i j}\right] G_{N}(d \underline{v}) & =\int \exp \left[\frac{\sigma}{N} \sum_{i \neq j} v_{i} v_{j} \bar{F}_{i j}\right] G_{N}(d \underline{v}) \\
& =\exp \left[\frac{1}{2} \log \operatorname{det}\left|I-\frac{2 \sigma}{N} F\right|\right],
\end{aligned}
$$

where $\bar{F}$ is the matrix $\bar{F}_{i j}$ for $i \neq j$ and $\bar{F}_{i i}=0$ with $\bar{F}_{i j}=\frac{1}{2}\left(F_{i j}+F_{j i}\right)$. Since $\bar{F}_{i j}$ is bounded it is easy to obtain the estimate (note $\operatorname{tr} \bar{F}=0$ )

$$
\log \operatorname{det}\left|I-\frac{2 \sigma}{N} \bar{F}\right| \leqq C \frac{\sigma^{2}}{N^{2}} \operatorname{Tr}(\bar{F})^{2} \leqq C \cdot \sigma^{2},
$$

provided $\sigma$ is suitably small. We therefore obtain

$$
\begin{aligned}
& \underset{N \rightarrow \infty}{\limsup } \frac{1}{N} \int \sum_{i \neq j} v_{i} v_{j} F_{i j}(\underline{x}) \hat{f}_{N}(\underline{x}, \underline{v}) d \mu_{N} \\
& \quad \leqq C \sigma \text { for all small } \sigma>0 \\
& \quad=0 .
\end{aligned}
$$

Again since the argument is insensitive to sign we have

$$
\limsup _{N \rightarrow \infty}\left|\frac{1}{N} \int \sum_{i \neq j} v_{i} v_{j} F_{i j}(\underline{x}) \hat{f}_{N}(\underline{x}, \underline{v}) d \mu_{N}\right|=0 .
$$

Combining (4.4) with (4.5) and (4.6) we get

$$
\begin{gathered}
\lim _{N \rightarrow \infty} E^{\hat{f}_{N}}\left[\sum_{i, j} 2 V^{\prime}\left(N\left(x_{i}-x_{j}\right)\right) \Phi\left(\omega_{N}^{0}\right) g\left(N x_{i}\right)\right. \\
\left.-\frac{1}{N} \sum \frac{\partial}{\partial x_{j}}\left(\Phi\left(\omega_{N}^{0}\right) g\left(N x_{j}\right)\right)\right]=0 .
\end{gathered}
$$

Since the function $\Phi(\omega)$ can be used just as well to cut off configurations having too many particles, we get from (4.7)

$$
E^{v}\left[\sum_{i, j} 2 V^{\prime}\left(y_{i}-y_{j}\right) \Phi\left(\omega^{0}\right) g\left(y_{i}\right)-\sum \frac{\partial}{\partial y_{i}}\left(\Phi\left(\omega^{0}\right) g\left(y_{i}\right)\right)\right]=0
$$

for any $v$ which is a limit point. But (4.8) is enough to guarantee that $v \in \Gamma_{l+1 / \varepsilon}^{(1)}$.

To see this we choose the function $\Phi(\omega)$ to be of the form $\psi_{1}\left(\omega_{\Lambda_{l}^{c}}\right) \psi_{2}\left(\omega_{\Lambda_{l}}\right)$, where $\psi_{1}$ and $\psi_{2}$ are functions of the configurations in the interior of $\Lambda_{l}=[-l, l]$ and its exterior $\Lambda_{l}^{c}$. We can choose $\psi_{2}\left(\omega_{\Lambda_{l}}\right)$ to be of the form

$$
\begin{aligned}
\psi_{2}\left(\omega_{\Lambda_{l}}\right) & =\phi\left(y_{1}, \ldots, y_{n}\right) \quad \text { if }\left|\omega_{\Lambda_{l}}\right|=n \\
& =0 \text { otherwise, }
\end{aligned}
$$


where $\left|\omega_{A_{l}}\right|$ is the cardinality of the configuration inside $[-l, l]$ and $y_{1}, \ldots, y_{n}$ are the locations of the $n$ particles inside $[-l, l]$. Here $\phi\left(y_{1}, \ldots, y_{n}\right)$ is a smooth symmetric function of $y_{1}, \ldots, y_{n}$. Although such a $\psi_{2}\left(\omega_{A_{l}}\right)$ is not smooth on configuration space it is easily approximated by smooth ones. There is no difficulty because the probability of finding a particle on the boundary is zero under $v$. If we denote by $v=v_{\Lambda_{l}}\left(d y_{1}, \ldots, d y_{n} \mid \omega_{\Lambda_{i}}, n\right)$ the conditional distribution of the configuration on $[-l, l]$ given the configuration on $\omega_{A_{l}}^{c}$ and the cardinality $\left|\omega_{A}\right|$ of $\omega_{A}$, then

$$
\begin{gathered}
\int\left\{\sum_{i=1}^{n} \sum_{y_{j}^{\prime} \in \omega_{\Lambda_{l}}} 2 V^{\prime}\left(y_{i}-y_{j}^{\prime}\right) \phi\left(y_{1}, \ldots, y_{n}\right) g\left(y_{i}\right)\right. \\
\left.-\sum_{i=1}^{n} \frac{\partial}{\partial y_{i}}\left(\phi\left(y_{1}, \ldots, y_{n}\right) g\left(y_{i}\right)\right)\right\} d v^{\prime}=0 .
\end{gathered}
$$

This identifies $v^{\prime}$ as the canonical Gibbs measure.

Proof of Theorem 4.3. For any configuration $x \in S^{N}$ we have associated the empirical measure

and smoothed version

$$
\alpha_{N}(d x)=\frac{1}{N} \sum \delta_{x_{i}}(d x)
$$

$$
\varrho_{\lambda}(x)=\frac{\lambda}{N} \sum_{i=1}^{N} h\left(\lambda\left(x-x_{i}\right)\right)
$$

We have to prove that $\varrho_{\lambda}(x)$ for $\lambda$ large but fixed and $\varrho_{\varepsilon N}(x)$ with $\varepsilon$ small but fixed are close. This amounts to proving that $\varrho_{\epsilon N}(x)$ does not have any oscillations. Just as in [4] we calculate the Young measures associated with $\varrho_{\varepsilon N}(\cdot)$ and show that as $N \rightarrow \infty$ and $\varepsilon \rightarrow 0$, these converge to a degenerate measure for almost every configuration. Corresponding to any density $\varrho(x)$ we define a measure $\pi$ on $S \times R^{+}$ by

Clearly

$$
\int F(x, \varrho) \pi(d x, d \varrho)=\int_{S} F(x, \varrho(x)) d x .
$$

$$
\begin{aligned}
& \int \varrho \pi(d x, d \varrho)=\int \varrho(x) d x=1, \\
& \int F(x) \pi(d x, d \varrho)=\int F(x) d x .
\end{aligned}
$$

If we denote by $\mathscr{M}$ the space of probability measures on $S \times R^{+}$, then through the map $\varrho(\cdot) \rightarrow \pi$ we can map the random density

$$
\varrho_{\varepsilon N}(x)=\left(h_{\varepsilon N} * \alpha_{N}\right)(x)
$$

into $\mathscr{M}$. If we consider the pair $\alpha_{N}$ and $\pi$ we get a map of $S^{N}$ into $M_{1}(S) \times \mathscr{M}$. We start with our basic $\hat{f}_{N} d \mu_{N}$ on $S^{N}$ and denote by $\hat{Q}_{N, \varepsilon}$ the induced distribution on $M_{1}(S) \times \mathscr{M}$. In order to establish Theorem 4.3, the main step, after establishing compactness of $\hat{Q}_{N, \varepsilon}$ as $N \rightarrow \infty$ and then as $\varepsilon \rightarrow 0$, is to show

Theorem 4.6. Let $\hat{Q}$ be any limit point of $\hat{Q}_{N, \varepsilon}$ as $N \rightarrow \infty$ and then as $\varepsilon \rightarrow 0$. Then for almost all $(\alpha, \pi)$ with respect to $\hat{Q}$

$$
\begin{aligned}
\alpha(d x) & =\varrho(x) d x, \\
\pi(d x, d \varrho) & =d x \delta_{\varrho(x)}(d \varrho)
\end{aligned}
$$

for some function $\varrho(x) \in L_{1}(S)$. 
We will prove Theorem 4.6 by means of the following lemmas.

Lemma 4.7. Let $\hat{Q}$ be the limit measure as above. Then

$$
\begin{gathered}
\hat{Q}\left[\alpha: \alpha(d x)=\varrho(x) d x \text { for some } \varrho \in L_{1}(S)\right]=1, \\
E^{\hat{Q}}\left[\int_{S} \varrho^{2}(x) d x\right] \leqq C \cdot B,
\end{gathered}
$$

where $C$ is a universal constant and $B$ is the bound on entropy.

Proof. Identical to Lemma 7.7 in [4].

Lemma 4.8. Let $\hat{Q}_{\varepsilon}$ be any limit measure of $\hat{Q}_{N, \varepsilon}$ as $N \rightarrow \infty$. Then

$$
\begin{gathered}
\hat{Q}_{\varepsilon}\left[(\alpha, \pi): \int \varrho \pi(d x, d \varrho)=1\right]=1, \\
\hat{Q}_{\varepsilon}\left[(\alpha, \pi): \pi(d x, d \varrho)=d x \pi_{x}(d \varrho)\right]=1, \\
\hat{Q}_{\varepsilon}\left[(\alpha, \pi): \alpha(d x)=\varrho(x) d x, \quad \text { with } \quad \varrho(x)=\int \varrho \pi_{x}(d \varrho)\right]=1 .
\end{gathered}
$$

Proof. Since this lemma is based only on entropy bounds, like Lemma 4.7 this proof is identical to Lemma 7.8 of [4].

It remains to prove that as $\varepsilon \rightarrow 0$ the limit points of $\hat{Q}_{\varepsilon}$ are supported by degenerate Young measures. Consider a function $g$ which is nonnegative, smooth, symmetric with compact support in $\left[-\frac{1}{4}, \frac{1}{4}\right]$ and having $\int g(x) d x=1$. For every $N$, $\delta$, and $\lambda$ we define

$$
G_{\delta, N, \lambda}(x)=\int_{-\infty}^{x} g_{\delta, N, \lambda}(y) d y,
$$

where

$$
g_{\delta, N, \lambda}(x)=N \delta g(N \delta x)-\lambda g(\lambda x) .
$$

We note that $G_{\delta, N, \lambda}$ has small compact support on $R$ and is therefore well defined as a function on $S$ provided $N \delta \geqq 1$ and $\lambda \geqq 1$. We consider the following test function on $S^{N} \times R^{N}$ :

$$
W_{\delta, N, \lambda}(\underline{x}, \underline{v})=\frac{1}{N} \sum_{i=1}^{N} v_{i} \sum_{j \neq i} G_{\delta, N, \lambda}\left(x_{i}-x_{j}\right) .
$$

We then have by calculation

$$
\begin{aligned}
\frac{1}{N^{2}} \sum_{i, j} & \left\{2 \sum_{k \neq i} N V^{\prime}\left(N\left(x_{i}-x_{k}\right)\right) \sum_{j \neq i} G_{\delta, N, \lambda}\left(x_{i}-x_{j}\right)\right. \\
& \left.-\sum_{l} v_{i} v_{l} \frac{\partial}{\partial x_{l}}\left(\sum_{j \neq i} G_{\delta, N, \lambda}\left(x_{i}-x_{j}\right)\right)\right\} \\
= & -W_{\delta, N, \lambda}(\underline{x}, \underline{v})+\frac{1}{N^{2}} L_{N} W_{\delta, N, \lambda} .
\end{aligned}
$$


We integrate by parts and use Schwartz' inequality to obtain

$$
\begin{aligned}
&\left|\int W_{\delta, N, \lambda}(\underline{x}, \underline{v}) \hat{f}_{N}(x, v) d \mu_{N}\right|=\left|\frac{1}{N} \int \sum v_{i} \sum_{j \neq i} G_{\delta, N, \lambda}\left(x_{i}-x_{j}\right) \hat{f}_{N}(\underline{x}, \underline{v}) d \mu_{N}\right| \\
&=\left|\int \frac{1}{N} \sum_{i=1}^{N} \frac{\partial \hat{f}_{N}}{\partial v_{i}} \sum_{j \neq i} G_{\delta, N, \lambda}\left(x_{i}-x_{j}\right) d \mu_{N}\right| \\
& \leqq\left(\int \frac{1}{N} \sum_{i=1}^{N}\left(\sum_{j \neq i} G_{\delta, N, \lambda}\left(x_{i}-x_{j}\right)\right)^{2} \hat{f}_{N} d \mu_{N}\right)^{1 / 2} \\
& \times\left(\int \frac{1}{N} \sum_{i=1}^{N} \frac{1}{\hat{f}_{N}}\left(\frac{\partial \hat{f}_{N}}{\partial v_{i}}\right)^{2} d \mu_{N}\right)^{1 / 2} \\
& \leqq \sqrt{\frac{C}{T}}\left(\int \frac{1}{N^{3}} \sum_{i=1}^{N}\left(\sum_{j \neq i} G_{\delta, N, \lambda}\left(x_{i}-x_{j}\right)\right)^{2} \hat{f}_{N} d \mu_{N}\right)^{1 / 2} \\
& \leqq \sqrt{\frac{C}{T}}\left(\int \frac{1}{N^{2}} \sum_{i, j} G_{\delta, N, \lambda}^{2}\left(x_{i}-x_{j}\right) \hat{f}_{N} d \mu_{N}\right)^{1 / 2} .
\end{aligned}
$$

From the definition of $\hat{f}_{N}$,

$$
\frac{1}{N^{2}} \int\left(L_{N} W_{\delta, N, \lambda}\right) \hat{f}_{N} d \mu_{N}=\frac{1}{N^{2} T}\left(\int W_{\delta, N, \lambda} \hat{f}_{N}^{T} d \mu_{N}-\int W_{\delta, N, \lambda} f_{N}^{0} d \mu_{N}\right),
$$

since $H\left(\hat{f}_{N}^{T}\right) \leqq H\left(\hat{f}_{N}^{0}\right) \leqq C N$, by Lemma 3.1 and Schwartz's inequality,

$$
\begin{aligned}
& \left|\int W_{\delta, N, \lambda} \hat{f}_{N}^{t} d \mu_{N}\right| \\
& \quad \leqq\left(\int \frac{1}{N} \sum_{i=1}^{N} v_{i}^{2} \hat{f}_{N}^{t} d \mu\right)^{1 / 2}\left(\int \frac{1}{N} \sum_{i=1}^{N}\left(\sum_{j \neq i} G_{\delta, N, \lambda}\left(x_{i}-x_{j}\right)\right)^{2} \hat{f}_{N}^{t} d \mu_{N}\right)^{1 / 2} \\
& \quad \leqq C_{1}\left(\int \frac{1}{N} \cdot N \cdot N^{2} \cdot C_{2}^{2} \cdot f_{N}^{t} d \mu_{N}\right)^{1 / 2} \\
& \quad \leqq C_{3} N .
\end{aligned}
$$

We have used here the fact that $G_{\delta, N, \lambda}$ is bounded by some $C_{2}$. Using (4.9), (4.10), and (4.11) it follows that

$$
\begin{gathered}
\lim _{\lambda \rightarrow \infty} \lim _{N \rightarrow \infty} \int \frac{1}{N^{2}} \sum_{i=1}^{N}\left\{2 \sum _ { k \neq i } V ^ { \prime } ( N ( x _ { i } - x _ { k } ) ) \left(\sum_{j \neq i} G_{\delta, N, \lambda}\left(x_{i}-x_{j}\right)\right.\right. \\
\left.\left.-\sum_{l=1}^{N} v_{i} v_{l} \frac{\partial}{\partial x_{l}}\left(\sum_{j \neq i} G_{\delta, N, \lambda}\left(x_{i}-x_{j}\right)\right)\right)\right\} \hat{f}_{N}(\underline{x}, \underline{v}) d \mu_{N}=0 .
\end{gathered}
$$

Let $A_{N}^{i j}(\underline{x})$ be the symmetric matrix

$$
\begin{aligned}
& {\left[A_{N}(\underline{x})\right]_{i j}=\frac{1}{N} g_{\delta, N, \lambda}\left(x_{i}-x_{j}\right) \text { for } i \neq j} \\
& =0 \quad \text { for } i=j \text {. }
\end{aligned}
$$


We can then use an argument similar to one used in Theorem 4.1 [Eqs. (4.7) and (4.8)] to get

$$
\begin{aligned}
& \int \frac{1}{N^{2}} \sum_{i \neq j} v_{i} v_{j} g_{\delta, N, \lambda}\left(x_{i}-x_{j}\right) \hat{f}_{N} d \mu_{N} \\
& \quad \leqq \frac{1}{\sigma} \int \hat{f}_{N}^{*}(\underline{x}) d \mu_{N}^{*}\left\{\frac{1}{2} \log \operatorname{det}\left[I-\frac{2 \sigma}{N} A_{N}(\underline{x})\right]\right\}+\frac{C}{2 T N \sigma} .
\end{aligned}
$$

Just as before we conclude that

$$
\lim _{N \rightarrow \infty}\left|\int \frac{1}{N^{2}} \sum_{i \neq j} v_{i} v_{j} g_{\delta, N, \lambda}\left(x_{i}-x_{j}\right) \hat{f}_{N} d \mu_{N}\right|=0 .
$$

We treat the remaining term in the same manner:

$$
\begin{aligned}
\int \frac{1}{N^{2}} & \sum_{i=1}^{N}\left(v_{i}^{2}-1\right) \sum_{j \neq i} g_{\delta, N, \lambda}\left(x_{i}-x_{j}\right) \hat{f}_{N} d \mu_{N} \\
\leqq & \frac{1}{\sigma} \int \hat{f}_{N}^{*}(x) d \mu_{N}^{*}\left\{\operatorname { l o g } \int \operatorname { e x p } \left[\frac{\sigma}{N^{2}} \sum_{i=1}^{N}\left(v_{i}^{2}-1\right)\right.\right. \\
& \left.\left.\times\left(\sum_{j \neq i} g_{\delta, N, \lambda}\left(x_{i}-x_{j}\right)\right)\right] G_{N}(d v)\right\}+\frac{C}{T N \sigma} .
\end{aligned}
$$

If we denote $\sum_{j \neq i} g_{\delta, N, \lambda}\left(x_{i}-x_{j}\right)$ by $\xi_{i}$ then $\left|\xi_{i}\right| \leqq C N^{2}$. Moreover

$$
\int \exp \left[\frac{\sigma \xi_{i}}{N^{2}}\left(v_{i}^{2}-1\right)\right] e^{-v_{i}^{2} / 2} \frac{1}{\sqrt{2 \pi}} d v_{i}=e^{-\frac{\sigma \xi_{i}}{N^{2}}-\frac{1}{2} \log \left(1-\frac{2 \xi_{i} \sigma}{N^{2}}\right)}
$$

Therefore for small $\sigma$,

$$
\begin{aligned}
& \log \int \exp \left[\frac{\sigma}{N^{2}} \sum_{i=1}^{N}\left(v_{i}^{2}-1\right) \xi_{i}\right] G_{N}(d v)=-\frac{\sigma}{N^{2}} \sum\left[\xi_{i}-\frac{1}{2} \log \left(1-\frac{2 \xi_{i} \sigma}{N^{2}}\right)\right] \\
& \quad \leqq-\frac{\sigma}{N^{2}} \sum \xi_{i}+\frac{\sigma}{N^{2}} \sum \xi_{i}+c_{1} \frac{\sigma^{2}}{2 N^{4}} \sum \xi_{i}^{2} \\
& \leqq c_{2} \sigma^{2} .
\end{aligned}
$$

We have now as before

$$
\lim _{N \rightarrow \infty}\left|\frac{1}{N^{2}} \int \sum_{i=1}^{N}\left(v_{i}^{2}-1\right) \sum_{j \neq i} g_{\delta, N, \lambda}\left(x_{i}-x_{j}\right) \hat{f}_{N} d \mu_{N}\right|=0 .
$$

If we combine (4.12), (4.13), and (4.14) we get

$$
\begin{aligned}
& \lim _{\lambda \rightarrow \infty} \lim _{N \rightarrow \infty} \mid \int \frac{1}{N^{2}} \sum_{i=1}^{N}\left\{\sum_{j, k \neq i} N V^{\prime}\left(N\left(x_{i}-x_{j}\right)\right) G_{\delta, N, \lambda}\left(x_{i}-x_{j}\right)\right. \\
& \left.-\sum_{j \neq i} g_{\delta, N, \lambda}\left(x_{i}-x_{j}\right)\right\} \hat{f}_{N} d \mu_{N} \mid=0 .
\end{aligned}
$$

From this point on the rest of the proof proceeds exactly like in [4]. First we note that with $\delta=1$ and $\lambda=1$,

$$
\sup _{N}\left|\int \frac{1}{N^{2}} \sum_{i=1}^{N}\left\{N V^{\prime}\left(N\left(x_{i}-x_{j}\right)\right) G_{1, N, 1}\left(x_{i}-x_{j}\right)\right\} \hat{f}_{N} d \mu_{N}\right| \leqq C .
$$


This provides us with the estimate

$$
\sup _{N}\left|\int \frac{1}{N} \sum_{i=1}^{N}\left\{\sum_{j=1}^{N} \psi\left(N\left(x_{i}-x_{j}\right)\right)\right\}^{2} \hat{f}_{N} d \mu_{N}\right| \leqq C .
$$

According to Lemma 7.9 of [4] this will give the required uniform integrability to take the limits as $N \rightarrow \infty$ and then $\lambda \rightarrow \infty$ and $\delta \rightarrow 0$. We remark that once $N \rightarrow \infty$, according to Lemma 4.5 we are in the local Gibbs situation and that puts us in the circumstances of [4]. In particular we get Lemma 7.10 of [4] establishing for any possible limit point the bound

$$
E^{Q} \int_{S} d x \int \varrho^{3} \pi_{x}(d \varrho) \leqq C
$$

\section{Hydrodynamic Limit}

We will prove in this section the main part namely (iv) for Theorem 1.1. Parts (i) and (ii) were essentially established in the previous section. We will prove (iii) in the next section. Of course we need some estimates to prove compactness. These will also be deferred to the next section.

For any function $J$ on $S$ which is smooth by Eq. (1.1) we have

$$
\frac{1}{N} \sum_{i=1}^{N} J\left(x_{i}(T)\right)-\frac{1}{N} \sum_{i=1}^{N} J\left(x_{i}(0)\right)=\int_{0}^{T} \sum_{i=1}^{N} J^{\prime}\left(x_{i}(t)\right) v_{i}(t) d t
$$

On the other hand

$$
\begin{aligned}
d\left(\sum_{i=1}^{N} J^{\prime}\left(x_{i}(t)\right) v_{i}(t)\right)= & N \sum_{i=1}^{N} J^{\prime \prime}\left(x_{i}(t)\right) v_{i}^{2}(t) d t \\
& -N \sum_{i=1}^{N}\left(2 \sum_{j \neq i} N V^{\prime}\left(N\left(x_{i}(t)-x_{j}(t)\right)\right)\right) J^{\prime}\left(x_{i}(t)\right) d t \\
& -\frac{N^{2}}{2} \sum_{i=1}^{N} J^{\prime}\left(x_{i}(t)\right) v_{i}(t) d t \\
& +N \sum_{i=1}^{N} J^{\prime}\left(x_{i}(t)\right) d w_{i}(t),
\end{aligned}
$$

we can rewrite this in the form

$$
\begin{aligned}
\int_{0}^{T} & \sum_{i=1}^{N} J^{\prime}\left(x_{i}(t)\right) v_{i}(t) d t=\frac{2}{N} \int_{0}^{T} \sum_{i=1}^{N} J^{\prime \prime}\left(x_{i}(t)\right) v_{i}^{2}(t) d t \\
& -\frac{2}{N} \int_{0}^{T} \sum_{i=1}^{N}\left(2 \sum_{j \neq i} N V^{\prime}\left(N\left(x_{i}(t)-x_{j}(t)\right)\right)\right) J^{\prime}\left(x_{i}(t)\right) c t \\
& +\frac{2}{N} \int_{0}^{T} \sum_{i=1}^{N} J^{\prime}\left(x_{i}(t)\right) d w_{i}(t) \\
& -\frac{2}{N^{2}}\left(\sum_{i=1}^{N} J^{\prime}\left(x_{i}(T)\right) v_{i}(T)-\sum_{i=1}^{N} J^{\prime}\left(x_{i}(0)\right) v_{i}(0)\right) .
\end{aligned}
$$


Combining it with (5.1)

$$
\begin{aligned}
\frac{1}{N} \sum J\left(x_{i}(T)\right)-\frac{1}{N} \sum J\left(x_{i}(0)\right) & \\
= & 2 \int_{0}^{T} F_{N}(t) d t+\frac{2}{N} \int_{0}^{T} \sum_{i=1}^{N} J^{\prime}\left(x_{i}(t)\right) d w_{i}(t) \\
& -\frac{2}{N^{2}}\left(\sum_{i=1}^{N} J^{\prime}\left(x_{i}(T)\right) v_{i}(T)-\sum_{i=1}^{N} J^{\prime}\left(x_{i}(0)\right) v_{i}(0)\right),
\end{aligned}
$$

where

$$
F_{N}(t)=\frac{1}{N} \sum_{i=1}^{N}\left\{v_{i}^{2}(t) J^{\prime \prime}\left(x_{i}(t)\right)-\sum_{j \neq i} 2 N V^{\prime}\left(N\left(x_{i}(t)-x_{j}(t)\right)\right) J^{\prime}\left(x_{i}(t)\right)\right\} \text {. }
$$

From (i) of Theorem 3.1 it is easy to show that

$$
\lim _{N \rightarrow \infty} E^{f_{N}^{0}}\left(\frac{2}{N^{2}}\left|\sum_{i=1}^{N} J^{\prime}\left(x_{i}(T)\right) v_{i}(T)-\sum_{i=1}^{N} J^{\prime}\left(x_{i}(0)\right) v_{i}(0)\right|\right)=0,
$$

and because $w_{1}(t), \ldots, w_{N}(t)$ are independent Brownian motions

$$
\lim _{N \rightarrow \infty} E^{f_{N}^{0}}\left|\frac{2}{N} \int_{0}^{T} \sum_{i=1}^{N} J^{\prime}\left(x_{i}(t)\right) d w_{i}(t)\right|=0 .
$$

The next step is to replace $v_{i}^{2}$ by 1 in (5.5).

\section{Lemma 5.1.}

$$
\lim _{N \rightarrow \infty} E^{f_{N}^{0}}\left(\int_{0}^{T}\left|\frac{1}{N} \sum J^{\prime \prime}\left(x_{i}(t)\right)\left(v_{i}^{2}(t)-1\right)\right| d t\right)=0 .
$$

Proof. By entropy inequality and logarithmic Sobolev inequality,

$$
\begin{aligned}
& \int\left|\frac{1}{4} \sum J^{\prime \prime}\left(x_{i}\right)\left(v_{i}^{2}-1\right)\right| \bar{f}_{N} d \mu_{N} \\
& \quad \leqq \int f_{N}^{*}(\underline{x}) d \mu_{N}^{*} \log \left\{\int \exp \left[\left|\frac{1}{N} \sum_{i=1}^{N}\left(v_{i}^{2}-1\right) J^{\prime \prime}\left(x_{i}\right)\right|\right] G_{N}(d \underline{v})\right\}+\frac{C}{2 N T} .
\end{aligned}
$$

By the law of large numbers

$$
\int \exp \left[\left|\frac{1}{N} \sum_{i=1}^{N}\left(v_{i}^{2}-1\right) J^{\prime \prime}\left(x_{i}\right)\right|\right] G_{N}(d \underline{v}) \rightarrow 1
$$

as $N \rightarrow \infty$, uniformly over $\underline{x}$. This proves the lemma.

Now the velocities are completely out of the picture and we only have to look at

$$
\int_{0}^{T} \frac{2}{N} \sum_{i=1}^{N}\left\{J^{\prime \prime}\left(x_{i}(t)\right)-\sum_{j \neq i} 2 N V^{\prime}\left(N\left(x_{i}(t)-x_{j}(t)\right)\right) J^{\prime}\left(x_{i}(t)\right)\right\} d t .
$$

We replace $J^{\prime}\left(x_{i}(t)\right)$ by $\frac{1}{2}\left[J^{\prime}\left(x_{i}(t)\right)-J^{\prime}\left(x_{j}(t)\right)\right]$, which we can, because of the skew symmetry of $V^{\prime}$. As in [4], we proceed to replace

$$
J^{\prime}\left(x_{i}(t)\right)-J^{\prime}\left(x_{j}(t)\right) \quad \text { by }\left(x_{i}(t)-x_{j}(t)\right) J^{\prime \prime}\left(x_{i}(t)\right)
$$


to get

$$
\int_{0}^{T} \frac{2}{N} \sum_{i=1}^{N} J^{\prime \prime}\left(x_{i}(t)\right)\left(1+\sum_{j=1}^{N} \psi\left(N\left(x_{i}(t)-x_{j}(t)\right)\right) d t\right.
$$

where

$$
\psi(z)=-z V^{\prime}(z)
$$

Now we can use Theorem 4.1 as in [4] and show that any weak limit will satisfy (iv) of Theorem 1.1.

\section{Some Auxiliary Lemmas}

Lemma 6.1. There exists a constant $C$ such that for all $N$,

$$
\int \frac{1}{N} \sum_{i=1}^{N}\left|\sum_{j=1}^{N} \psi\left(N\left(x_{i}-x_{j}\right)\right)\right|^{2} \bar{f}_{N} d \mu_{N} \leqq C .
$$

Proof. This is precisely (4.17).

Lemma 6.2 (Compactness). For every smooth $J(\cdot)$ on $S$, and $\varepsilon>0$,

$$
\lim _{\delta \rightarrow 0} \limsup _{N \rightarrow \infty} P^{f_{N}^{0}}\left[\sup _{\substack{0 \leq s<t \leq T \\|s-t| \leqq \delta}}\left|\left\langle J, \xi_{N}(t)\right\rangle-\left\langle J, \xi_{N}(s)\right\rangle\right| \geqq \varepsilon\right]=0 .
$$

Proof. According to Eq. (5.1),

$$
\begin{aligned}
\left\langle J, \xi_{N}(t)\right\rangle-\left\langle J, \xi_{N}(s)\right\rangle & =\frac{1}{N} \sum J\left(x_{i}(t)\right)-\frac{1}{N} \sum J\left(x_{i}(s)\right) \\
& =\int_{s}^{t} \sum_{i=1}^{N} J^{\prime}\left(x_{i}(\sigma)\right) v_{i}(\sigma) d \sigma .
\end{aligned}
$$

We can rewrite this using (5.3) as

$$
\begin{aligned}
\int_{s}^{t} \sum_{i=1}^{N} J^{\prime}\left(x_{i}(\sigma)\right) v_{i}(\sigma) d \sigma=\frac{2}{N} \int_{s}^{t} \sum_{i=1}^{N} J^{\prime \prime}\left(x_{i}(\sigma)\right) v_{i}^{2}(\sigma) d \sigma & \\
& -\frac{2}{N} \int_{s}^{t} \sum_{i=1}^{N} 2 \sum_{j \neq i} N V^{\prime}\left(N\left(x_{i}(\sigma)-x_{j}(\sigma)\right)\right) J^{\prime}\left(x_{i}(\sigma)\right) d \sigma \\
& +\frac{2}{N} \int_{s}^{t} \sum_{i=1}^{N} J^{\prime}\left(x_{i}(\sigma)\right) d \omega_{i}(\sigma) \\
& -\frac{2}{N^{2}}\left[\sum_{i=1}^{N} J^{\prime}\left(x_{i}(t)\right) v_{i}(t)-\sum_{i=1}^{N} J^{\prime}\left(x_{i}(s)\right) v_{i}(s)\right] \\
= & A_{1}(s, t)+A_{2}(s, t)+A_{3}(s, t)+A_{4}(s, t), \\
A_{1}(s, t)= & \frac{2}{N} \int_{s}^{t} \sum_{i=1}^{N} J^{\prime \prime}\left(x_{i}(\sigma)\right)\left[v_{i}^{2}(\sigma)-1\right] d \sigma+\frac{2}{N} \int_{s}^{t} \sum J^{\prime \prime}\left(x_{i}(\sigma)\right) d \sigma, \\
\left|A_{1}(s, t)\right| \leqq & 2 \int_{0}^{T}\left|\frac{1}{N} \sum J^{\prime \prime}\left(x_{i}(\sigma)\right)\left(v_{i}^{2}(\sigma)-1\right)\right| d \sigma+2|t-s|\left\|J^{\prime \prime}\right\|_{\infty} .
\end{aligned}
$$


Since $E^{f_{N}^{0}}\left[\int_{0}^{T}\left|\frac{1}{N} \sum J^{\prime \prime}\left(x_{i}(\sigma)\right)\left(v_{i}^{2}(\sigma)-1\right)\right| d \sigma\right] \rightarrow 0$ as $N \rightarrow \infty$ it follows that

$$
\begin{gathered}
\lim _{\delta \downarrow 0} \varlimsup_{N \rightarrow \infty} P^{f^{0}}\left[\sup _{\substack{0 \leq s \leq t \leq T \\
|\bar{t}-s| \leqq \delta}}\left|A_{1}(s, t)\right| \geqq \varepsilon\right]=0, \\
A_{2}(s, t)=-\frac{2}{N} \int_{s}^{t} \sum_{i=1}^{N} N V^{\prime}\left(N\left(x_{i}(\sigma)-x_{j}(\sigma)\right)\right)\left(J^{\prime}\left(x_{i}(\sigma)\right)\right) d \sigma,
\end{gathered}
$$

and

$$
\begin{aligned}
\left|A_{2}(s, t)\right| & \leqq \frac{2}{N}\left\|J^{\prime \prime}\right\|_{\infty} \int_{s}^{t} \sum_{i, j} \psi\left(N\left(x_{i}(\sigma)-x_{j}(\sigma)\right)\right) d \sigma \\
& \leqq(t-s)^{1 / 2} \cdot \frac{2}{N}\left\|J^{\prime \prime}\right\|_{\infty}\left[\int_{s}^{t}\left(\sum_{i, j} \psi\left(N\left(x_{i}(\sigma)-x_{j}(\sigma)\right)\right)\right)^{2} d \sigma\right]^{1 / 2} \\
& \leqq(t-s)^{1 / 2} 2\left\|J^{\prime \prime}\right\|_{\infty}\left(\int_{0}^{T} \frac{1}{N} \sum_{i=1}^{N}\left[\sum_{j \neq i} \psi\left(N\left(x_{i}(\sigma)-x_{j}(\sigma)\right)\right)\right]^{2} d \sigma\right)^{1 / 2} .
\end{aligned}
$$

From Lemma 6.1

$$
\lim _{\delta \rightarrow 0} \limsup _{N \rightarrow \infty} P^{f_{N}^{0}}\left[\sup _{\substack{0 \leq s \leq t \leq T \\ \mid \bar{t}-\bar{s} \leqq \delta}}\left|A_{2}(s, t)\right| \geqq \varepsilon\right]=0 .
$$

From Doob's inequality

$$
P^{f_{N}^{0}}\left[\sup _{0 \leqq t \leqq T}\left|\frac{2}{N} \int_{0}^{t} \sum_{i=1}^{N} J^{\prime}\left(x_{i}(\sigma)\right) d \omega_{i}(\sigma)\right| \geqq \varepsilon\right] \leqq \frac{2}{\varepsilon^{2}} \cdot \frac{4}{N^{2}} \cdot N \cdot\left\|J^{\prime}\right\|_{\infty}^{2} .
$$

Therefore

$$
\lim _{N \rightarrow \infty} P^{f_{N}^{0}}\left[\sup _{0 \leqq s \leqq t \leqq T}\left|A_{3}(s, t)\right| \geqq \varepsilon\right]=0 .
$$

In order to handle $A_{4}(s, t)$ it is sufficient to prove

$$
\lim _{N \rightarrow \infty} P^{f_{N}^{0}}\left[\sup _{0 \leqq t \leqq T} \frac{1}{N^{2}}\left|\sum_{i=1}^{N} J^{\prime}\left(x_{i}(t)\right) v_{i}(t)\right| \geqq \varepsilon\right]=0 .
$$

Let us denote by $\eta_{N}(t)=\sum_{i=1}^{N} J^{\prime}\left(x_{i}(t)\right) v_{i}(t)$. We can rewrite (5.2) in the form

$$
\begin{aligned}
d \eta_{N}(t)= & -\frac{N^{2}}{2} \eta_{N}(t) d t \\
& +N \sum_{i=1}^{N} J^{\prime \prime}\left(x_{i}(t)\right) v_{i}^{2}(t) d t \\
& -N \sum_{i=1}^{N}\left(2 \sum_{j \neq i} N V^{\prime}\left(N\left(x_{i}(t)-x_{j}(t)\right)\right) J^{\prime}\left(x_{i}(t)\right)\right), d t \\
& +N \sum_{i=1}^{N} J^{\prime}\left(x_{i}(t)\right) d \omega_{i}(t) .
\end{aligned}
$$


Integrating this we get

$$
\begin{aligned}
\eta_{N}(t)= & \eta_{N}(0) e^{-N^{2} t / 2} \\
& +N^{2} \int_{0}^{t} A_{N}(s) e^{-\left(N^{2} / 2\right)(t-s)} d s \\
& +N^{2} \int_{0}^{t} B_{N}(s) e^{-\left(N^{2} / 2\right)(t-s)} d s \\
& +N^{2} \int_{0}^{t} e^{-\left(N^{2} / 2\right)(t-s)} d M_{N}(s)
\end{aligned}
$$

where

$$
\begin{aligned}
A_{N}(s) & =\frac{1}{N} \sum J^{\prime \prime}\left(x_{i}(s)\right) v_{i}^{2} \\
\left|B_{N}(s)\right| & \leqq \frac{1}{N}\left\|J^{\prime \prime}\right\|_{\infty} \sum_{i, j} \psi\left(N\left(x_{i}(s)-x_{j}(s)\right)\right), \\
M_{N}(s) & =\frac{1}{N} \int_{0}^{s} \sum J^{\prime}\left(x_{i}(\sigma)\right) d \omega_{i}(\sigma) .
\end{aligned}
$$

Since we have a uniform bound on

$$
\begin{aligned}
& E^{f_{N}^{0}}\left[\left|\eta_{N}(0)\right|\right] \leqq \log \int \exp \left[\left|\sum J^{\prime}\left(x_{i}\right) v_{i}\right|\right] d \mu_{N}+C N \\
& \leqq C^{\prime} N, \\
& P^{f_{N}^{0}}\left[\frac{1}{N^{2}} \sup _{0 \leqq t \leqq T}\left|\eta_{N}(0) e^{-N 2 t / 2}\right| \geqq \delta\right] \rightarrow 0 \text { as } \quad N \rightarrow \infty .
\end{aligned}
$$

In order to establish (6.1) we need only prove

$$
\begin{aligned}
& P^{f_{N}^{0}}\left[\sup _{0 \leqq t<T} \int_{0}^{t} e^{-\left(N^{2} / 2\right)(s-t)}\left|A_{N}(s)\right| d s \geqq \varepsilon\right] \rightarrow 0 \text { as } \quad N \rightarrow \infty, \\
& P^{f_{N}^{0}}\left[\sup _{0 \leqq t<T} \int_{0}^{t} e^{-\left(N^{2} / 2\right)(s-t)}\left|B_{N}(s)\right| d s \geqq \varepsilon\right] \rightarrow 0 \text { as } \quad N \rightarrow \infty,
\end{aligned}
$$

and

$$
\begin{gathered}
P^{f_{N}^{0}}\left[\sup _{0 \leqq t<T}\left|\int_{0}^{t} e^{-\left(N^{2} / 2\right)(s-t)} d M_{N}(s)\right| \geqq \varepsilon\right] \rightarrow 0 \text { as } \quad N \rightarrow \infty \\
\left|A_{N}(s)\right| \leqq\left|\frac{1}{N} \sum J^{\prime \prime}\left(x_{i}(s)\right)\right|+\left|\frac{1}{N} \sum J^{\prime \prime}\left(x_{i}(s)\right)\left(v_{i}^{2}(s)-1\right)\right| \\
\leqq\left\|J^{\prime \prime}\right\|_{\infty}+\left|\frac{1}{N} \sum J^{\prime \prime}\left(x_{i}(s)\right)\left(v_{i}^{2}(s)-1\right)\right|
\end{gathered}
$$


Clearly,

$$
\begin{gathered}
\sup _{0 \leqq t \leq T} \int_{0}^{T} e^{-\left(N^{2} / 2\right)(t-s)} d s \rightarrow 0 \text { as } N \rightarrow \infty, \\
\sup _{0 \leqq t \leqq T} \int_{0}^{T} e^{-\left(N^{2} / 2\right)(t-s)}\left|\frac{1}{N} \sum J^{\prime \prime}\left(x_{i}(s)\right)\left(v_{i}^{2}(s)-1\right)\right| d s \\
\leqq \int_{0}^{T}\left|\frac{1}{N} \sum J^{\prime \prime}\left(x_{i}(s)\right)\left(v_{i}^{2}(s)-1\right)\right| d s,
\end{gathered}
$$

and since $E^{f_{N}^{0}}\left[\int_{0}^{T}\left|\frac{1}{N} \sum J^{\prime \prime}\left(x_{i}(s)\right)\left(v_{i}^{2}(s)-1\right)\right| d s\right] \rightarrow 0$ as $N \rightarrow \infty$ we have (6.4).

We estimate

$$
\begin{aligned}
& \int_{0}^{t} e^{-\left(N^{2} / 2\right)(t-s)}\left|B_{N}(s)\right| d s \leqq\left(\int_{0}^{t} e^{N^{2}(t-s)} d s\right)^{1 / 2}\left(\int_{0}^{t}\left|B_{N}(s)\right|^{2} d s\right)^{1 / 2} \\
& \leqq \frac{1}{N}\left(\int_{0}^{T}\left|B_{N}(s)\right|^{2} d s\right)^{1 / 2} \\
& \int_{0}^{T}\left|B_{N}(s)\right|^{2} d s \leqq \frac{1}{N} \int_{0}^{T} \sum_{i=1}^{N}\left(\sum_{j=1}^{N} \psi\left(N\left(x_{i}(s)-x_{j}(s)\right)\right)\right)^{2} d s .
\end{aligned}
$$

Since we have a uniform bound on the expectation of the right-hand side from Lemma 6.1 we have (6.5).

Finally we turn to (6.6). Let us pretend $N^{2} T$ is an integer. Then

$$
\begin{aligned}
& \sup _{0 \leqq t \leqq T}\left|e^{-\left(N^{2} / 2\right) t} \int_{0}^{t} e^{\left(N^{2} / 2\right) s} d M_{N}(s)\right| \\
& \quad \leqq \sup _{1 \leqq k \leqq N^{2} T} e^{-\left(N^{2} / 2\right)(k-1) / N^{2}} \sup _{0 \leqq t \leqq k / N^{2}}\left|\int_{0}^{t} e^{N^{2} s / 2} d M_{N}(s)\right| .
\end{aligned}
$$

We can estimate

$$
\begin{aligned}
P^{f_{N}^{0}} & {\left[\sup _{0 \leqq t \leqq T}\left|e^{-N^{2} t / 2} \int_{0}^{t} e^{\left(N^{2} / 2\right) s} d M_{N}(s)\right| \geqq \varepsilon\right] } \\
& \leqq \sum_{k=1}^{N^{2} T} P^{f_{N}^{0}}\left[\sup _{0 \leqq t \leqq k / N^{2}}\left|\int_{0}^{t} e^{-\left(N^{2} / 2\right) s} d M_{N}(\varepsilon)\right| \geqq \varepsilon e^{(k-1) / 2}\right] \\
& \leqq \sum_{k=1}^{N^{2} T} \frac{e^{-(k-1)}}{\varepsilon^{2}} E^{f_{N}^{0}}\left[\int_{0}^{k / N^{2}} e^{\left(N^{2} / 2\right) s} d M_{N}(s)\right]^{2} \\
& \leqq \sum_{k=1}^{N^{2} T} \frac{1}{\varepsilon^{2}} \cdot e^{-(k-1)} e^{k}\left\|J^{\prime}\right\|_{\infty}^{2} \cdot \frac{1}{N^{3}} \\
& =\frac{T}{N} \cdot e \cdot\left\|J^{\prime}\right\|_{\infty}^{2} \cdot \frac{1}{\varepsilon^{2}}
\end{aligned}
$$

$\rightarrow 0$ as $N \rightarrow \infty$ and we are done.

Lemma 6.3. Let $Q$ be any limit point of the distributions of $\xi_{N}(\cdot)$ under $P^{f_{N}^{0}}$ as $N \rightarrow \infty$, where $f_{N}^{0}$ satisfies $H_{N}\left(f_{N}^{0}\right) \leqq C N$. Then

$$
E^{Q}\left(\int_{0}^{T} d t \int_{S} d \theta \frac{1}{q(\theta, t)}\left[\frac{\partial}{\partial \theta} P(q(\theta, t))\right]^{2}\right) \leqq \frac{C}{2}
$$

with the same constant. 
Proof. Given two integers $n$ and $l$ we consider a family of functions $u_{1}(x), \ldots, u_{n}(x)$, $F_{1}(x), \ldots, F_{l}(x)$ which are smooth functions on $S$. We consider also a family $g_{1}\left(y_{1}, \ldots, y_{l}\right), \ldots, g_{n}\left(y_{1}, \ldots, y_{l}\right)$ of smooth functions with compact support on $R^{l}$. We use these functions to define a family $U_{1}, \ldots, U_{n}$ of functions on $S^{N} \times R^{N}$ and $G_{1}, \ldots, G_{n}$ on $S^{N}$

$$
\begin{aligned}
U_{r}(\underline{x}, \underline{v}) & =\sum_{j=1}^{N} v_{j} u_{r}\left(x_{j}\right), \\
G_{r}(\underline{x}) & =g_{r}\left(\frac{1}{N} \sum_{j=1}^{N} F_{1}\left(x_{j}\right), \ldots, \frac{1}{N} \sum_{j=1}^{N} F_{l}\left(x_{j}\right)\right) .
\end{aligned}
$$

By a direct calculation for each $r$,

$$
\begin{gathered}
\frac{1}{N} \sum_{i=1}^{N}\left\{2 \sum_{j=1}^{N} N V^{\prime}\left(N\left(x_{i}-x_{j}\right)\right) u_{r}\left(x_{i}\right)-v_{i}^{2} u_{r}^{\prime}\left(x_{i}\right)\right\} \\
=-\frac{1}{2} U_{r}(\underline{x}, \underline{v})-\frac{1}{N^{2}} L_{N} U_{r}(x, v) .
\end{gathered}
$$

We want to prove first that

$$
\lim _{N \rightarrow \infty} \frac{1}{N^{2}} \int\left(L_{N} U_{r}\right)(\underline{x}, \underline{v}) \cdot G_{r}(\underline{x}) \bar{f}_{N} d \mu_{N}=0 .
$$

In fact since $G_{r}(\underline{x})$ is only a function of $\underline{x}$ and $L_{N}$ is first order in $x$ derivatives,

$$
\left(L_{N} U_{r}\right) G_{r}=L_{N}\left(U_{r} G_{r}\right)-U_{r}\left(L_{N} G_{r}\right)
$$

and

$$
\frac{1}{N^{2}} \int L_{N}\left(U_{r} G_{r}\right) \bar{f}_{N} d \mu_{N}=\frac{1}{N^{2} T}\left[\int U_{r} G_{r} f_{N}^{T} d \mu_{N}-\int U_{r} G_{r} f_{N}^{0} d \mu_{N}\right]:_{1}
$$

Since both $f_{N}^{T}$ and $f_{N}^{0}$ satisfy entropy bounds,

$$
\begin{gathered}
H_{N}\left(f_{N}\right) \leqq C N, \\
\left|\int U_{r} G_{r} f_{N} d \mu_{N}\right| \leqq\left\|G_{r}\right\|\left\|U_{r}\right\| \sqrt{N}\left(\int \sum v_{i}^{2} f_{N} d \mu_{N}\right)^{1 / 2} \\
\leqq C^{\prime} N,
\end{gathered}
$$

where $f_{N}$ can be either $f_{N}^{T}$ or $f_{N}^{0}$. Therefore

$$
\lim _{N \rightarrow \infty} \frac{1}{N^{2}} \int L_{N}\left(U_{r} G_{r}\right) \bar{f}_{N} d \mu_{N}=0 .
$$

Moreover

$$
\frac{1}{N^{2}} \int U_{r}\left(L_{N} G_{r}\right) \bar{f}_{N} d \mu_{N}=\frac{1}{N^{2}} \int \sum_{i, j} v_{i} v_{j} u_{r}\left(x_{i}\right) \frac{\partial G_{r}}{\partial x_{j}}(\underline{x}) \bar{f}_{N} d \mu_{N} .
$$

By the arguments we have used in the proof of Theorem 4.6 we can replace $v_{i} v_{j}$ by $\delta_{i j}$ and because it is obvious that

$$
\lim _{N \rightarrow \infty} \frac{1}{N^{2}} \int \sum_{r=1}^{N} u_{r}\left(x_{i}\right) \frac{\partial G_{r}}{\partial x_{i}}(\underline{x}) \bar{f}_{N} d \mu_{N}=0,
$$


we conclude that

$$
\lim _{N \rightarrow \infty} \frac{1}{N^{2}} \int U_{r}\left(L_{N} G_{r}\right) \bar{f}_{N} d \mu_{N}=0
$$

(6.8) follows immediately from (6.9) and (6.10).

We now turn to the relation (6.7). Let us use $\bar{f}_{N}$ to construct a measure $\bar{Q}_{N}$ on $M_{1}(S)$ by the map $\left(x_{1}, \ldots, x_{N}\right) \rightarrow \frac{1}{N}\left(\delta_{x_{1}}+\ldots \delta_{x_{N}}\right)$ and let $\bar{Q}$ be any weak limit. According to the results of Sects. 4 and 5,

$$
\lim _{N \rightarrow \infty} \int \frac{1}{N} \sum_{i=1}^{N}\left\{2 \sum_{j=1}^{N} N V^{\prime}\left(N\left(x_{i}-x_{j}\right)\right)\left(u_{r}\left(x_{i}\right)-u_{r}^{\prime}\left(x_{i}\right)\right)\right\} G_{r}(\underline{x}) \bar{f}_{N} d \mu_{N}
$$

along the subsequence giving the weak limit $\bar{Q}$ can be represented as

$$
-E^{\bar{Q}}\left\{\int P(q(x)) u_{r}^{\prime}(x) G_{r}\left(\int F_{1}(x) q(x) d x, \ldots, \int F_{l}(x) q(x) d x\right)\right\} .
$$

If we now use (6.7) and (6.8) and sum over $r=1,2, \ldots$, we obtain

$$
\begin{aligned}
&\left|E^{\bar{Q}}\left\{\int P(q(x)) \sum_{r=1}^{n} u_{r}^{\prime}(x) G_{r}\left(\int F_{1}(x) q(x) d x, \ldots, \int F_{l}(x) q(x) d x\right) d x\right\}\right| \\
& \leqq \limsup _{N \rightarrow \infty}\left|\frac{1}{2} \int \sum_{r=1}^{n} U_{r}(\underline{x}, \underline{v}) G_{r}(\underline{x}) \bar{f}_{N} d \mu_{N}\right| \\
&= \limsup _{N \rightarrow \infty}\left|\frac{1}{2} \int \sum_{r=1}^{n} \sum_{i=1}^{N} u_{r}\left(x_{i}\right) G_{r}(\underline{x}) v_{i} \bar{f}_{N} d \mu_{N}\right| \\
&= \underset{N \rightarrow \infty}{\limsup }\left|\frac{1}{2} \int \sum_{r=1}^{n} \sum_{i=1}^{N} u_{r}\left(x_{i}\right) G_{r}(\underline{x}) \frac{\partial \bar{f}_{N}}{\partial v_{i}} d \mu_{N}\right| \\
& \leqq \limsup _{N \rightarrow \infty} \frac{1}{2}\left(\int \frac{1}{\bar{f}_{N}} \sum_{i=1}^{N}\left(\frac{\partial \bar{f}_{N}}{\partial v_{i}}\right)^{2} d \mu_{N}\right)^{1 / 2} \\
& \times\left(\int \sum_{i=1}^{N}\left(\sum_{r=1}^{n} u_{r}\left(x_{i}\right) G_{r}(\underline{x})\right)^{2} \bar{f}_{N} d \mu_{N}\right)^{1 / 2} \\
& \leqq \limsup _{N \rightarrow \infty} \frac{1}{2}\left(\frac{2 C}{N T}\right)^{1 / 2} N^{1 / 2}\left(\int \frac{1}{N} \sum_{i=1}^{N}\left(\sum_{r=1}^{n} u_{r}\left(x_{i}\right) G_{r}(\underline{x})\right)^{2} \bar{f}_{N} d \mu_{N}\right)^{1 / 2} \\
&= \frac{1}{2}\left(\frac{2 C}{T}\right)^{1 / 2}\left(E^{Q} \int q(x) d x\left(\sum_{r=1}^{n} u_{r}(x) G_{r}\left(\int F_{1}(x) q(x) d x, \ldots, \int F_{l}((x) q(x) d x)\right)\right)^{1 / 2} .\right.
\end{aligned}
$$

Denoting by $\omega$ the typical point in $M_{1}(S)$ we can rewrite (6.11) as

$$
\left|E^{\bar{Q}} \int P(q(x)) \frac{\partial \hat{G}}{\partial x}(x, \omega) d x\right| \leqq\left(\frac{C}{2 T}\right)^{1 / 2}\left(E^{\bar{Q}} \int \hat{G}^{2}(x, \omega) q(x) d x\right)^{1 / 2}
$$

for test functions $\hat{G}$ from a suitable class. First we have it for $\hat{G}$ of the form

$$
\sum u_{r}(x) G_{r}\left(\left\langle F_{1}, q\right\rangle, \ldots,\left\langle F_{l}, q\right\rangle\right) .
$$


Then we can obtain the inequality for $\hat{G}$ of the form

$$
\sum u_{r}(x) \hat{G}_{r}(\omega) \text {. }
$$

The next step is to use test functions of general form $\hat{G}(x, \omega)$ but with reasonable bounds on $\partial \hat{G} / \partial x$. Then by standard regularization techniques one can prove

$$
E^{\bar{Q}} \int \frac{1}{q(x)}\left(\frac{\partial P(q(x))}{\partial x}\right)^{2} d x \leqq \frac{C}{2 T} .
$$

It is easy to see that if $Q$ is a weak limit on $C\left[[0, T] ; M_{1}(S)\right]$ then $\bar{Q}$ is just the average marginal distribution over the time interval $[0, T]$. Therefore

$$
\begin{aligned}
E^{Q} \int_{0}^{T} \int \frac{1}{q(x, t)}\left(\frac{\partial}{\partial x} P(q(x, t))\right)^{2} d x d t & \leqq \frac{C}{2 T} \cdot T \\
& =\frac{C}{2}
\end{aligned}
$$

Lemma 6.4. There is at most one weak solution of the equation

$$
\frac{\partial \varrho(t, x)}{\partial t}=2(P(\varrho(t, x)))_{x x}
$$

with initial condition $\varrho(0, x)=\varrho_{0}(x)$ among the class of nonnegative solutions satisfying

$$
\begin{gathered}
\int_{0}^{T} \int \varrho^{3}(t, x) d t d x<\infty \\
\int_{0}^{T} \int\left(\frac{\partial P(\varrho(t, x))}{\partial x}\right)^{2} \frac{1}{\varrho(t, x)} d t d x<\infty .
\end{gathered}
$$

Proof. This has been carried out in [4].

\section{References}

1. Deuschel, J.D., Stroock, D.W.: Large deviations. New York: Academic Press 1989

2. De Masi, A., Presutti, E.: Lectures on the collective behavior of particle systems. C.A.R.R. Reports in Mathematical Physics, 5/89, 1989

3. Papanicolaou, G., Varadhan, S.R.S.: Ornstein-Uhlenbeck process in a random potential. Commun. Pure Appl. Math. 35, 819-834 (1985)

4. Varadhan, S.R.S.: Scaling limits for interacting diffusions. Commun. Math. Phys. 135, 313-353 (1991)

Communicated by J. L. Lebowitz 